\title{
Study of Respiratory Tissue Engineering Model for Viral Infection and Bacterial Microbiome Interaction Under Physiological and Pathological Condition
}

\section{Ryan Septa Kurnia $^{1 *}$ and Radiana Dhewayani Antarianto ${ }^{2}$}

${ }^{1}$ Doctoral Program in Biomedical Science, Faculty of Medicine Universitas Indonesia, Jakarta; ${ }^{2}$ Departements of Histology, Faculty of Medicine, Universitas Indonesia, Jakarta.

\begin{abstract}
Respiratory epithelium is one of the mucosal epithelial which functions as protective and adaptive barrier against continuously inhaled substances including pathogens and allergens. Current research about mechanism of disease and drug development is conducted in 2D cell culture or in animal models. The 2D cell culture models poorly imitate the condition in vivo and provide limited utility due to mimic tissue physiology in multicellular organisms. Although animal models can be used as pre-clinical tools for new-agent screening prior to clinical testing, there is a growing awareness of the limitations of animal research and its inability to make reliable predictions for human clinical trials. Recently, 3D cell culture produced by researchers to improve better mimic tissue physiology of better in vitro cell culture model. It is may enable microbiologists to create infection models that combine respiratory tissue culture engineering with the virus-relevant complexity of in vivo models. Mechanism of infection disease have shown that the normal microbiota in host health has remarkably improved our understanding of the interactions between microbiota and invading pathogens. Commensal bacteria as microbiome can potentially influence mechanism of disease either hindering or promoting the viral infection and sometimes aggravate the disease. In this review, we discuss the histology of respiration epithelium and mucous layers, various microbiome that contribute to the interaction between the host and microbes against infection on the mucus layer in top of the respiratory epithelium, and technology that can be developed in tissue engineering techniques for reconstruction to mimic the microenvironment in respiratory tissue engineering.
\end{abstract}

Received | December 07, 2020; Accepted | February 13, 2021; Published | February 23, 2021

*Correspondence | Ryan Septa Kurnia, Doctoral Program in Biomedical Science, Faculty of Medicine Universitas Indonesia, Jakarta; Email: ryanseptakurnia@gmail.com

DOI | https://dx.doi.org/10.17582/journal.hv/2021/8.1.8.22

Citation | Kurnia, R.S. and R.D. Antarianto. 2021. Study of respiratory tissue engineering model for viral infection and bacterial microbiome interaction under physiological and pathological condition. Hosts and Viruses, 8(1): 8-22.

Keywords: Respiratory, Microbiome, Tissue, Engineering, Model

\section{Introduction}

A cute respiratory disease by viral infections are the leading cause of respiratory disease worldwide. The severity of this disease may vary markedly from asymptomatic to mild and gradually progressed into severe wheezing, bronchitis, and pneumonia. Unlike other pathogens that restricted to tropical areas, the respiratory viruses are distributed globally and efficiently transmitted from person to person (Eugenia et al., 2013; Proença-Módena et al., 2011; Troy and Bosco, 2016). In the last twenty years, several viral epidemics such as current novel coronavirus outbreak (now called SARS-CoV-2, causing the disease Covid-19), MERS-CoV in 2012, H1N1 influenza pandemic in 2009, and SARS-CoV in 2002-2003 
have been recorded (Abrahao and de Arruda, 2020). Emerging of new virus are often associated with migration, deforestation, and other anthropogenic activities. This can trigger the emergence of several new diseases due to the eviction of wild animals from nature which is a reservoir of various zoonotic viruses. Accurate condition of viral infection mechanism in a laboratory setting is needed for identification of pathogen-host interaction and potential therapeutic drug development (Guan et al., 2020; Zhou et al., 2020).

Recent studies on the mechanism of virus infection and drug development research, in-vitro models with $2 \mathrm{D}$ cell cultures and in-vivo with animal models are widely used. In 2D culture, the cells were grown in monolayer condition thus offering unnatural growth kinetics and attachments. Representation of cells microenvironment can be lacking because the cell's natural microenvironment is not fully representative (Saji et al., 2019). Animal models can be used as preclinical tools for new-agent screening and understand how viruses replicate in individuals. It is important for virologists to unravel the basic mechanisms of virus pathogenesis inside the individuals. Selection of the suitable animal models which represents similar anatomy and physiology with human is important for the goal of the study (Adachi and Miura, 2014; Han et al., 2018; Swearengen, 2018). Animal models have several limitations e.g failure to accurately mimic the human disease progressions, lack of best practice standards for animal testing, and physiological differences that have an impact on variations in the molecular target homology (Mak et al., 2014).

Nowadays, tissue engineering recently proposed for the design of reliable in vitro models of healthy or pathological tissues and organs (Caddeo et al., 2017). Tissue engineering in a form of 3D cell culture receive more attention as they exhibit protein expression patterns and intracellular junctions that are similar in vivo states compared to classic monolayer cultures (Jaroch et al., 2018). This model can more faithfully recapitulate the biology of specific tissue and can be used for longer periods. Many of these models are still relatively simple, the culture of cells at an air-liquid interface or culture of two different cell types separated by a porous membrane. However, increasingly complex and sophisticated models of tissues and organs are being engineered and modified to create unique disease models in a condition that closely imitates in vivo microenvironment could be elaborately constructed in vitro (Benam et al., 2015; Chaicharoenaudomrung et al., 2019; Ramanan et al., 2014).

A balanced host-microbe interaction is necessary for maintaining homeostasis (Parker etal.,2018). Bacterial microbiome in human demonstrates inter-individual variability, and can be influenced by the environment, genetics, and diet. Recent study shows that host's normal microbiome can potentially influence viral infections either hindering or promoting the viral infection and sometimes aggravate the disease (Lima et al., 2019; Wilks et al., 2013). It significantly contributes toward protection against pathogens by competing for shared nutrients and niches or through enhancing host defense mechanisms (Kamada et al., 2013). Additionally, the use of tissue engineering model to study the respiratory viral infection and microbiome interaction has not been reviewed extensively. The purpose of this review is to describe the state of art co-culture of 3D epithelial respiration in tissue engineering construction to determine microbiota dysbiosis in respiratory tract and viral infection mechanism model. This review will discuss the histology of respiration epithelium and mucous layers, various microbiome that contribute to the interaction between the host and microbes against infection on the mucus layer in top of the respiratory epithelium, and technology that can be developed in tissue engineering techniques for reconstruction to mimic the microenvironment in respiratory tissue engineering.

\section{Histology of respiratory tract epithelium}

The respiratory system subdivides into a conducting and respiratory portion. The conducting portion deliver air to the respiratory tissue and is characterized by rigid walls that consist keep the airways open. This portion starts from the nasal cavity, pharynx, larynx, tracheal and bronchial tree. Respiratory portion located in the pulmonary alveoli, that the portion of this system where the gas exchange between blood and air occur. Alveoli are the most unique part because the microenvironment is the boundary between the air-filled space and the surfactant bordering the epithelium basal membrane that fused with a very tight capillary endothelial basal membrane (Krause, 2005).

Conduction portion of respiratory tract starts from 
the nasal cavity, pharynx, and larynx that is covered by ciliated pseudostratified columnar epithelium with goblet cell, forms a continuous lining of the airway lumen homeostasis (Scherzad et al., 2019; Yuksel and Turkeli, 2017). Pharynx is part of the conduction zone of respiratory system and digestive system. The pharyngeal phase serves to protect the airway during swallowing, a coordinated physiological response to prevent respiration during swallowing which lasts about 0.5 to 1.5 seconds. Swallowing mechanism itself serves as a vital protector of the airway and voluntary actions of over 30 nerves and muscles produce this coordinated movement (Panara and Padalia, 2020). The upper part of the pharynx, the nasopharynx extends from the base of the skull to the upper surface of the soft palate. This includes the space between the internal nares and the soft palate and is located above the oral cavity. In areas subject to abrasion, a nonkeratinizing stratified squamous epithelium may occur, such as on the edge of the soft palate and posterior wall of the pharynx, where these surfaces make contact during swallowing (Krause, 2005).

Larynx is part of the respiratory tract in the form of a tubular segment with primarily cartilaginous held together by a series of ligament and membrane that connects the pharynx and trachea. Internally, the laryngeal muscles move components of the larynx provides a protective sphincter at the inlet of the air passages and is responsible for phonation and breathing. The upper pair constitutes vocal cords (vestibular folds), covered by the respiratory epithelium beneath which lie numerous serous glands within the lamina propria. The anterior surface and about half the posterior surface of the epiglottis are covered by nonkeratinized stratified squamous epithelium. The vocal cords also are covered by a wet stratified squamous epithelium, but elsewhere the larynx is lined by the respiratory passage type of epithelium (Krause, 2005). Larynx does contain immunological tissue in the form of epithelial follicles (larynx-associated lymphoid tissue: LALT) in an age and disease dependent manner. This immunological activity is likely to be crucial for determining the development and outcome of infectious, inflammatory disease and neoplastic disease (Barker et al., 2006).

Trachea is part of respiratory tract consists of incomplete rings of hyaline cartilage and smooth muscle that embedded into a fibrous membrane of elastic connective tissue (Brand and Schafer, 2014).
These cartilages give the trachea rigidity and prevent its collapse. It extends from the lower part of the larynx to its bifurcation into two main bronchi. The average adult trachea measures about $11 \mathrm{~cm}$ in length with a diameter that varies from 2 to $2.5 \mathrm{~cm}$. The pediatric trachea is smaller, more deeply placed, and more mobile. Tracheal luminal mucosa of lined by pseudostratified columnar respiratory epithelium consisting of ciliated and clara cells. The proportion of clara cells is increase in the caudal part of the trachea, and goblet cells that rests on a thick basal lamina are only found rarely (Fernandez et al., 2008; Navarro et al., 2017). In addition, goblet cells secrete mucus that contain many defensive compounds into the mucosal fluid, including mucins, antibodies, immunoglobulin A, lysozymes, histatins, nitric oxide and lactoferrin to protect the host actively (Binsker et al., 2020; Patel, 2017). Both goblet cells and submucosal glands produce mucus, which forms a gel layer on the epithelial surface of the respiratory tract to act as a protective barrier against the external environment by trapping particulate matter, including pathogens. Trapped matter can then be expelled from the airways by the rhythmic beating of cilia bundles on the airway epithelium by cough (Aghapour et al., 2018; Zanin et al., 2016).

The bronchial structure is a continuation and branching of the trachea that allow air to be transported from the surrounding atmosphere to the lung. This structure is similar histologically to the trachea that are lined with ciliated pseudostratified columnar epithelium. The term bronchial tree is the large airways in the distal trachea that form secondary or lobar bronchi, then these branches supply the individual lung lobes and are connected to segmental or tertiary bronchi, which supply individual lung segments. The cartilaginous structure decrease with decreasing bronchial diameter. The muscularis develops into the rings of smooth muscle encircling bronchi and bronchioles. As a bronchial diameter becomes smaller, the epithelium becomes flatter and the number of secretory cells decrease with no longer have submucosal glands or cartilage. The epithelium becomes simple columnar, with clara cell replacing mucous cell and basal cells disappearing. Bronchioles end in alveolar duct, which contain spiral smooth muscle and are lined by alveolar epithelium (Chanez and Bourdin, 2008; Haschek et al., 2002; Reynolds et al., 2015).

Pulmonary alveoli are the most specialized part 
of the respiratory system and is responsible for its most important function, gas exchange. The microenvironment of the structure is the boundary between the air-filled space and the surfactant which is bordered by a thin layer of epithelium (pneumocytes/ type I alveolar epithelial cells) and cuboid-shaped epithelium (pneumocytes/ type II alveolar epithelial cells). The basal epithelial membrane of this structure fuses into the capillary endothelial basal membrane with high tight junctions. The design of the interalveolar septum that contain of smooth muscle cells, connective tissue/ fibers and elastin with complex structures providing a large surface area, a thin diffusion barrier, requirements of stability to preventing overdistension or collapse of alveoli, and also flexibility. The mechanism of $\mathrm{O}_{2}$ and $\mathrm{CO}_{2}$ gas exchange occurs by diffusion in alveolar membranes. Oxygen from the alveoli diffuses freely through the cells to capillaries for eventual distribution by the systemic blood flow (Alvarado and Arce, 2016; Crystal et al., 2008).

\section{Mechanism of microbes infection in respiratory tract}

In the respiratory tract mechanism of infection, complex interactions occur between pathogens (microorganisms) and epithelial cells that lining the respiratory tract. The airways epithelium is the primary site for viral infection and replication. The entry of viruses into respiratory epithelium cells must first penetrate the mucous layer, which provides the first line of defense against invading pathogens (Scherzad et al., 2019; Yuksel and Turkeli, 2017). In addition, respiratory tract epithelium consist goblet and clara cells that secrete many defensive compounds into the mucosal fluid, including mucins, antibodies, defensins, protegrins, collectins, cathlecidins, lysozyme, histatins, and nitric oxide. Oxidants, such as nitric oxide that is produced following the induction of nitric oxide synthase-2 (NOS-2), have also been shown to inhibit viral infections (Aghapour et al., 2018; Zanin et al., 2016).

High viral replication is generally known in the proximal trachea and nasopharyngeal epithelium. Increased immune cell infiltration, CD8 + cytotoxic $\mathrm{T}$ cells in tracheal epithelial cells are a form of immune response occurring during infection. Virus-specific cytotoxic (cell killing) $\mathrm{T}$ cells are the first line of adaptive immunity responsible for elimination of infected cells. Pathological abnormalities of infected trachea are often marked with reddened and swollen mucosal surfaces. Hemorrhagic tracheitis and bronchitis were observed in 50\% of influenza cases (Deist et al., 2017; Lambert et al., 2016; Taubenberger and Mores, 2008). Generally, viral infection will cause innate and adaptive immune response to facilitates viral clearance. At the same time, it causes excessive inflammation and results in tissue damage (Yoo et al., 2013).

The entry of the virus in the host epithelial cell is strongly influenced by the receptors contained in the cell. For example, seasonal Influenza A virus binds to $\mathrm{AS} \alpha 2-6 \mathrm{Gal}$ receptor, which are found primarily on the surface of epithelial cells in the nasal mucosa, tracheal and bronchi. In contrast, highly pathogenic avian $\mathrm{H} 5 \mathrm{~N} 1$ virus binds to $\mathrm{AS} \alpha 2-3 \mathrm{Gal}$ receptor, which is found on alveolar epithelial cells (Troy and Bosca, 2016). The interaction between influenza virus and mucus was first identified in studies conducted in the 1940s and 1950s through observing receptordestroying enzymes from Vibrio cholerae that can reduce the inhibitory action of mucoproteins over time in a manner similar to influenza viruses. The enzyme has the ability as a hydrolytic cleavage of glycosidic bonds of neuraminic acid groups (sialic acid) to D-galactose or D-galactosamine, so it is called neuraminidase or sialidase. This proves that neuraminidase plays an important role in determining whether the virus can penetrate the mucous barrier to infect the underlying cells. Neuraminidase enzyme may facilitate infection by cleaving sialic acid on mucin layer to prevent viruses from becoming trapped before they infect the epithelium (Zanin et al., 2016).

Underneath the mucus layer of the cells there are glycocalyx, dense forest of highly diverse glycoproteins and glycolipids. The glycocalyx, which is atop the epithelial cells, is a fuzzy and filamentous coat that is weakly acidic and consists of sulfated mucopolysaccharides. The glycocalyx has numerous functions, including structural roles in proteins, protein folding and mediation of self-recognition by the immune system (Linden et al., 2008). Therefore, the interaction of pathogens with the glycocalyx is very important in the viral lifecycle. However, respiratory mucus also contains sialic acids and glycoproteins, and the various virus interaction with these is also important. High sialic acid in mucosal surface results in a strongly negative surface charge, increasing the rigidity of the polymer via charge repulsion (Zanin et al., 2016). Sialic acid naturally occurs in prokaryotes as nine-carbon keto sugar acids derived 
from N-acetylneuraminic acid (Neu5Ac). Sialidase (sialoglycosidase) that found in viruses, bacteria, protozoa, fungi, and metazoans is major enzyme that facilitate interaction between the host and pathogen by cleaving the sialic acid from sialoglycoproteins (Figure 1). This could expose that receptors for enzymatic interactions and ligand binding by contributing to biological functions, such as cellular interaction and conformational stabilization of glycoproteins in the cell membranes (Sudhakara et al., 2019).

Bacterial colonization of the respiratory tract also has a similar mechanism to be able to reach the surface of the epithelium, the bacteria must penetrate the mucous layer to gain access to epithelial cell. There are several pathogenic opportunistic factors in the airway including transient association with mucus, weak association with host carbohydrates, and strong association with host surface proteins (Siegel and Weiser, 2015). Bacterial virulence factors are molecules that influence between bacteria and host cell and/or substance, including processes such as adherence to cell membrane, colonization, and invasion. Several virulence factors mechanism is likely used to reach epithelial cell, including motility and digestion of the mucus layer by bacterial enzyme (sialogycosidase). Bacterial virulence factors act as protease, lipase, toxins, adhesion factors, biofilm, capsules made of carbohydrate and antiphagocytic factors. Bacterial capsule is known for their role in limiting optimization with complement and antibodies, also contributing to avoid mucus-mediated clearance. These factors allow bacteria to "escape" and interact with epithelial cell. For example, bacteria that sialoglycoidase enzyme can penetrate the mucus layer and reach the target cell by digest the mucus layer with their enzyme and consume oligosaccharides such in the mucus layer as a carbon source. The balance between host and bacterial colonization can be tipped in either direction by a range of factors that modify each stage of attachment, growth, and immune evasion (Zachary and McGavin, 2017).
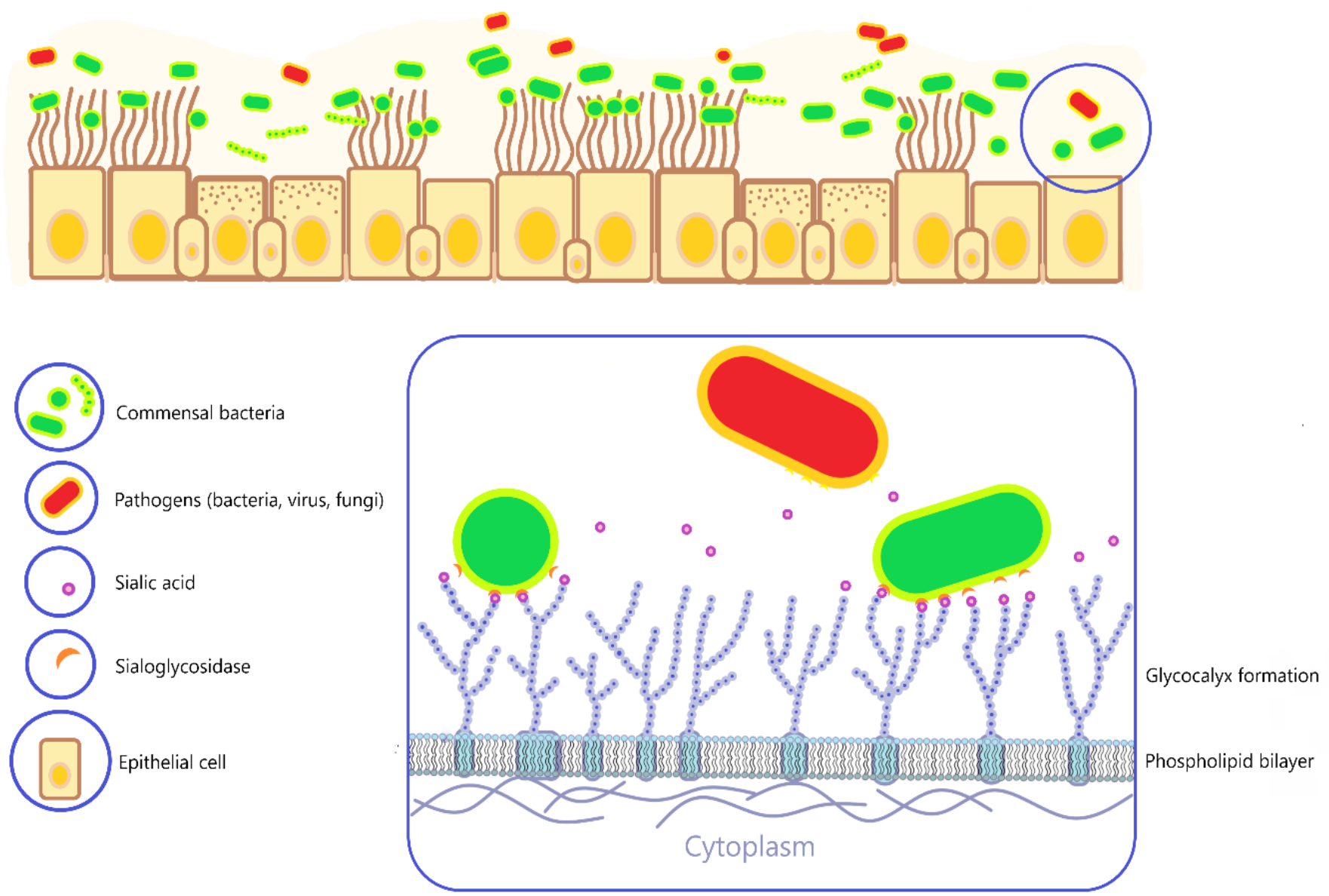

Figure 1: Physiological condition of respiratory tract microbiome illustration. Diverse commensal bacteria in the respiratory tract live with a balanced composition without pathological conditions. Various pathogens (bacteria, viral and fungal) with relatively limited numbers are less likely to infect the host if there is no drastic changes in conditions. Glycocalyx is glycoprotein and glycolipid that cover the surface of the host epithelial cell membrane. Sialic acid which is in the structure has a place to attach to several normal microorganisms and pathogens which are assisted by sialoglycosidase. 
Respiratory mucosal tissue microbiome

Over the last decade, human microbiome has become a hot topic since the launch of National Institutes of Health (NIH) Human Microbiome Project and the International Human Microbiome Consortium. This is seen as a new insight to understanding human health and disease by determining human microbiome (Neto et al.,2017). Microbiome is comprised of the collective genome of microorganisms (bacteria, archaea, viruses, and fungi) that symbiotic and pathogenic, living in and on all vertebrates (Taneja, 2017). It also revealed that the lungs have a diverse microbial community, which were previously considered sterile in health. This new concept has challenged numerous longheld assumptions regarding respiratory health and the pathogenesis of respiratory disease (Dickson et al., 2016). The entire surface of the respiratory tract is inhabited by niche-specific bacterial communities. Along with the anatomical development of the respiratory tract, the formation of respiratory microorganisms is considered to have an influence on the process of morphogenesis and regulation of the immune system (Man et al., 2017; Wilks et al., 2013).

Human upper respiratory structure is a combination between the outer skin surface and the mucosa that is in direct and continuous contact with the outside world. Exposure to these external environmental conditions causes the formation of ecological conditions in various microbial communities (de Steenhuijsen et al., 2015). The upper respiratory tract breathes at least 70001 of air with an average number of microorganisms $10^{4}-10^{6}$ bacterial cells per cubic meter of air inhaled per day. There are five of the most common genera of bacteria found in nasopharynx, including Moraxella, Staphylococcus, Corynebacterium, Streptococcus, and Dolosigranulum (Figure 2).
Commensal bacteria can positively interact with the host and each other by colonizing the upper airways. Interaction of competition usually occurs between commensal and opportunistic pathogens to fight over nutrients in the airways of freely glucose and iron in limited quantities (Wang et al., 2018; Kumpitsch et al., 2019).

In the pharynx, the most common microbiome found include Prevotella, Capnocytophaga, Campylobacter, Veillonella, Streptococcus, Neisseria and Haemophilus. Some species of bacteria such as Streptococcus pneumonia, Staphylococcus aureus, Haemophilus influenza and Mycoplasma pneumonia are known to be pathogenic, however they can adapt to the pharyngeal environment, rendering the host asymptomatic (Gao et al., 2014). Whereas in the laryngeal area several other bacteria that cause respiratory infection are found including Chlamydia, Bordetella, Aggregatibacter, Pseudomonas, and Bacteroides (Gong et al., 2013). Some of these variation in microbiota diversity and bacterial composition are often associated with a risk of laryngeal carcinoma and vocal cord polyps (Gong et al., 2017; Wang et al., 2019).

Microbiome that lives in the lower respiratory tract plays an important role against micro-environmental homeostasis that serves as a defense against potential invading viral infection. Generally, some bacteria that are often found in lower respiratory tract microbiome in healthy individuals include Prevotella, Veillonella, Streptococcus, Fusobacterium, and Hemophilus (Gu et al., 2019). In the trachea microbiome bacteria reflects membership from both the oral cavity and upper airways. The anatomic location of the trachea that connects the upper and lower respiratory tract is the reason it can occur. Some of the microbiome that

\section{Bacterial community}

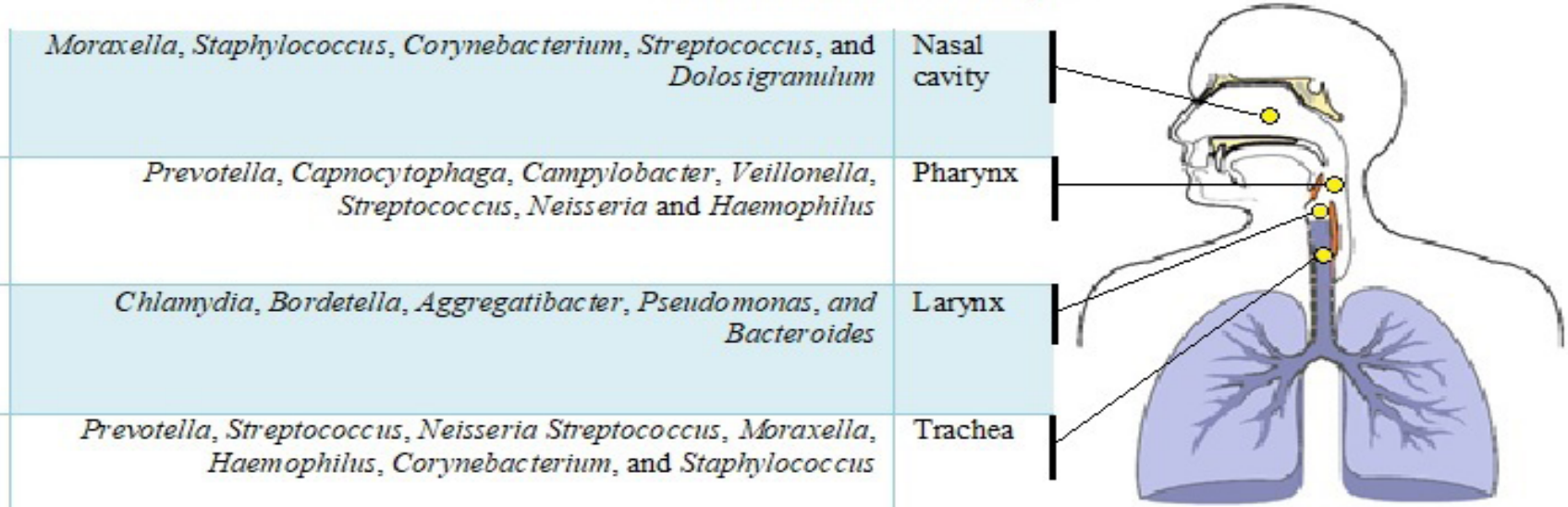

Figure 2: Bacterial community along the nasal cavity, pharynx, larynx, and trachea. 
found in trachea are Prevotella, Streptococcus, Neisseria, Streptococcus, Moraxella, Hemophilus, Corynebacterium, and Staphylococcus (Perez-Losada et al., 2017, 2018).

The composition and alteration of the microbiome community in the bronchial and lung are often associated with asthma, chronic obstructive pulmonary disease (COPD) and cystic fibrosis (CF). In healthy subject, microbiome composed of oropharynx bacteria which migrate to bronchial tree through aspiration. Some of these bacteria are Streptococcus and Veillonella that are found in the bronchial mucosa at low loads. Changes in the microenvironmental conditions cause modification of bacterial composition by overgrowth of specific bacteria. In pathological conditions such as asthma, COPD, and CF, high load of several bacteria such as Pseudomonas, Hemophilus and Legionella are found (Garcia et al., 2014; Millares et al., 2017). Local $\mathrm{pH}$, blood flow, temperature, epithelial cells, oxygen levels, and disposition of inflammatory cells are some conditions that affect the proliferation and dynamic change of the microbial diversity of the respiratory tract (O'Dwyer et al., 2016).

\section{$3 D$ tissue engineered upper respiratory tract epithelium to} investigate early infection

The development of in vitro models to determine the interaction of microbes and host cells begins with observation using a hostcell that is grown in a monolayer 2-D flat. This approach advances our understanding of the mechanisms underlying infection and disease in host cells. However, it is experiencing problems due to differences between in-vivo and in-vitro conditions which cause changes in the original behavior of host cells and microbes. Some of these differences are more dominant in microenvironment conditions including oxygen tension, extracellular matrix (ECM), homotypic or heterotypic cells surrounding the single cell, cytokines, hormone, microbiota composition/ localization, and biomechanical forces (e.g., fluid shear, stretch, compression) (Barrila et al., 2018; Barthes et al., 2014). Therefore, natural microenvironments of monolayer 2-D flat cells are not fully represented. Recently, 3-D cell cultures in tissue engineering are used to improve better in vitro cell culture models that resemble in vivo conditions in multicellular organisms (Saji et al., 2019). This model can mimic the nature of tissues and show changes in viability, morphology, differentiation status, proliferation capacity, and gene expression profiles. One of the benefits is to find out the mechanism of viral infection and the impact that occurs in vitro on tissue-specific hosts, innate immune function, cell cycle status, and polarity resembling in vivo. In order to get the appropriate tissue engineering model, several things to consider include the selection of scaffold and extracellular matrix (ECM), cell type, medium, biomechanical forces to form microenvironment (Barrila et al., 2018; Ramanan et al., 2014).

\section{Construction of 3D microenvironment of upper respiratory tract}

Construction models that can be used to mimic the upper airway epithelium begin with twodimensional (2D) monolayers, differentiated airway pseudostratified cell layers either submerged or at the air-liquid interface (ALI) (between 2D and 3D) and 3D cell culture models. In general, there are several main components for tissue engineering construction, including scaffold to support cell attachment and growth, cells with which to populate the scaffold, and bioreactors that provide the nutrients needed by developed tissue, waste removal, and other physical and biochemical stimuli to generate organized and functional tissue (Calle et al., 2017). The choice of scaffold is most often derived from collagen, to mimic the natural extracellular matrix or, a decellularized biomimetic structure or tissue can be used as a substrate for cell culture. The choice of substrate is important because it can affect cell adhesion, polarity, cell differentiation and barrier formation (De Rudder et al., 2018).

\section{Scaffold for respiratory tract tissue engineering}

The complex nature of respiratory organs requires the development of hybrid scaffolding that has more than one material to be able to resemble in-vivo. The mechanism and interaction between pathogens and hosts in the development of tissue engineering is focused on respiratory epithelium based on three basic approaches: in situ homing (adhesion, proliferation, and support migration to cells); cell sheet technology (release intact cell sheets with deposited ECM); and fluid interface techniques (promote epithelial differentiation into multi-lineage phenotypes) (Kumar et al., 2017). Scaffold material commonly applied in the development of respiratory tissue engineering consists of natural polymers (collagen), synthetic polymeric materials (Poly-capcaprolactone), and composites of natural synthetic combination materials (Dekorin-gelatin-PCL). Most research on tissue engineering to date has used primary 
tracheobronchial or alveolar epithelial cells which are mixed cell populations containing endogenous progenitors that may contribute to proliferation in 3D construction (O'Leary et al., 2015).

Natural polymers are known to originate from the decellularization process of organs or tissues, so they can form ECM with low antigenicity and minimal risk of allograft rejection in regenerative medicine. Collagen, chitosan, and gelatin are natural scaffolds that can provide bioactive properties to interact with cells. The limitation of this scaffold is the lack of tissue and organs for decellularization and low mechanical stability, while synthetic biomaterials can be produced at low cost, in large quantities and have a longer shelf life and provide more mechanical stability (Eltom et al., 2019; Khazraee et al., 2018). In addition to determining the material, the design and fabrication of scaffolding is also very important to mimic the shape of in-vivo respiratory tissue so that it has good biocompatibility, biodegradability and better cell viability (Dhasmana et al., 2020). Various fabrication techniques have been used in the past to produce different materials into scaffolding for tissue engineering purposes. These techniques include solvent casting and particle washing, gas foam, fiber meshes and fiber bonding, phase separation, melt molding, emulsion freeze drying, solution casting, freeze drying and bioprinting (Henkel and Hutmacher, 2013). Scaffold design research for artificial trachea with cylindrical structure with coverage with ciliated respiratory mucosa, and adequate cartilage has been carried out by combining the fabrication techniques of electrospun polycaprolactone (PCL) nanofibers (inner) and 3D-printed PCL microfibers (outer) (Kim et al., 2020).

\section{Cells for scaffold repopulation}

Development of human disease models in vitro often used cell lines and primary cells such as tracheal epithelial, alveolar epithelial and bronchial epithelial cell lines to better represent respiratory tissue because specific cell types can accurately recapitulate the phenotype of the disease (Estermann et al., 2020; Thiebes et al., 2015). Immortal cell lines that are often used to mimic the respiratory tract are 16HBE (human bronchial epithelial cells) and Calu3 and A549 (cells from human lung carcinoma). These cells have the advantages of easy acquisition, source reliability, and stable for repeat experiments. But these cells are dependent on the availability of tissue and are difficult to obtain, reproduce slowly, have a limited life span with the altered genotype compared to in vivo tissue, and the inability to obtain a desired disease phenotype. The development of tissue engineering using induced pluripotent stem cells (iPSC) provides an extraordinary opportunity to overcome this limitation. that is because of its ability to express several transcription factors and the resulting pluripotent cells can renew themselves indefinitely. The cell can also differentiate into several types of cells depending on the induction and the conditions given. The combination of different cell types within a model is a strategy accurately represent the host microenvironment and the interactions therein (Benam et al., 2015).

Basically the cells are seeded directly on the surface of the initial scaffold until a monolayer cell culture is obtained and then it can be removed, combined, and shaped in such a way as to obtain a $3 \mathrm{D}$ formation where the microenvironment conditions of the artificial tissue can be regulated (Mandrycky et al., 2017). Cells that seeded on the scaffold aim to deposit extracellular matrix components such as fibronectin and collagen. Research with the aim of modeling the interaction between host and pathogen with epithelial cells as physical barriers to pathogens that colonize the human respiratory tract its integrity is a key requirement. Parameters to consider in the formation of monolayer cells such as physiological epithelial function usually depend on the formation of tight junctions (TJs) that unite the epithelial cells that form a barrier. Expression of the Zonula occludens 1 (ZO-1) protein which is an important component of TJ can accurately depict inter-cellular contours on the apical side of the epithelial cell layer (Marrazzo et al., 2016).

\section{Biomechanical forces and bioreactor}

The architecture of respiratory tissue has very high complexity to be emulated in the form of $3 \mathrm{D}$ tissue engineering and has the same biomechanical properties of breathing. The complex structure consists of compartments throughout the network in a flexible and dynamic matrix that continues to move at the two cell layer interfaces, with a balanced combination of structural and functional conditions (Mandrycky et al., 2017). Mechanical properties and forces affect the modulation of cell fate, especially on stem cell differentiation. In 3-D scaffold, stem cells through the addition of growth factors and mechanical strength 
have been shown to induce desired cell differentiation and tissue formation (Roomans, 2010). In bronchial epithelium, mechanical forces in the form of bronchoconstriction as well as the cyclical stretching of the airway can have an impact to the luminal level by the presence of air flow and transduction of the cilia resulting in additional mucus. However, in addition, it can influence activation promoting airway remodeling that is influenced by ECM, increased epithelial properties such as solute transport, viral gene delivery, and secretion of ECM modifying proteins (Prakash et al., 2015).

Current bioreactors usually contain an integrated 3D cell construction, e.g., epithelium and endothelium; or epithelial and fibroblastic, or several types of cells that aim to mimic conditions in vivo. Conditions inside the bioreactor such as downward or cross flow forces, microgravity environments, rotating wall cell culture systems, fluid shear, compression, stretched and pressure loads are used to achieve dynamic conditions. This is done to stimulate the differentiation and proliferation of cells into tissue, especially in stem cells, known as mechano-differentiation (Selden and Fuller, 2018). Some research on the development of respiratory tissue techniques shows that mechanical stimulation in the form of air flow most often occurs in bioreactors. Controlled air flow in the bioreactor is known to contribute to the formation of tight junctions in confluent monolayers of lung epithelial cells. Additionally, the incorporation of perfusion and ventilation in the bioreactor of the airway allows maintenance of the survival of epithelial and endothelial cells (Panoskaltsis, 2015; Petersen et al., 2011; Poon et al., 2012). Mechanical stimuli such as water-liquid interfaces and ventilation produced by negative pressure are needed especially for the development of lung tissue. Measurement microfluidic parameters in real-time are also needed to provide complex bioprocesses that mimic the microenvironment of the lungs (Tebyanian et al., 2019).

\section{Tissue engineering for microbiome model}

Respiratory tissue engineering modeling to observe host and microbiome interaction in co-culture epithelial cells model in vitro offer the possibility to investigate if in a controlled environment with reduced complexity compared to in-vivo. It is therefore hoped that the respiratory tissue engineering model will not only focus on colonization with single species of microbe or probiotic but also can be used to monitor short-term host-microbe interactions, such as innate immune responses of the epithelial cells and physiological changes of bacterial and epithelial cells such as cytokine production and tight junction functionality. The first respiratory tissue engineering study in vitro model was done for bacterial biofilm formation on differentiated Calu-3 epithelial cells at ALI in co-culture with Haemophilus influenzae bacteria (Starner et al., 2006). Similar modeling mechanisms are also used to study Staphylococcus aureus colonization of airway epithelial cells and observations of physiological changes that occur during the colonization process. However, this causes the epithelial layer completely disrupted within 1 day after inoculation (Kiedrowski et al., 2016). The model demonstrates limitations in the time frame of host microbial culture and the ability to replicate the respiratory tract microenvironment in an appropriate manner due to cell toxicity, duration of microbial culture by interaction between epithelial culture cells and bacteria that are limited to hours or days. Based on these studies several things that need to be considered that will affect the success of the model, namely the number of microbes that are infected in cells ranging from $0.01-10$ bacteria / host cell. Flow washing in the bioreactor is also a matter to be considered to prevent over colonization from occurring, however, the failure of the host compartment due to inadequacy in mimicking the host microenvironment as the most frequent host cell due to undifferentiation or differentiated epithelial cell culture (De Rudder et al., 2018).

The most recent work on respiratory tissue engineering in $3 \mathrm{D}$ is a comprehensive human tracheobronchial mucosa from the pseudostratified epithelium of primary cells of human origin and the underlying stromal tissue. In this research, a model was developed that could mimic native tissue such as mucociliary differentiation from epithelial sheets and basement membrane formation. Furthermore, the addition of Haemophilus influenzae bacteria results in bacterial associations and crossing the mucous layer. these results can potentially be adjusted to study medium / long term host-pathogenic processes (Marrazzo et al., 2016). Other development of respiratory engineering is ALI model of human bronchioles with epithelial and endothelial tissue known as the lung-in-a-chip, developed based on microfluidic devices that mimic the microarchitecture and dynamic microenvironment 
of alveolar-capillary units of living human lungs. Soft lithography-based microfabrication is used for building a three-dimensional microchannel system in such a way as to obtain the formation of alveolarcapillary interfaces, human alveolar epithelial cells and pulmonary microvascular endothelial cells favored into the upper and lower chambers. This model is claimed to be used to observe inflammatory mechanisms, such as cytokines and proinflammatory bacteria, to the upper alveolar compartment which induces activation of endothelial cells on opposite sides of the membrane and increases the expression of their adhesion molecules (Huh et al., 2012; Huh, 2015). As in previous studies of microbiome in tissue engineering models were carried out in the intestinal tract using the intestine on a chip model, which at present is at an early stage of development microbial loads in the lumen. The model is used to observe the interaction of microbial and host-cell viability, gene-expression profiles during culture, mucusproducing human intestinal epithelial and complex community of living commensal gut microorganisms (Jalili et al., 2019; Poceviciute and Ismagilvo, 2019). In the 3D structure model, two popular transformed intestinal epithelial cells (IECs), Caco-2 and HT29 cells propagated in ECM such as Matrigel, an analog basement membrane that helps direct the polarity of the epithelium. Recently, intestinal pluripotent stem cells are also used in vitro and are known as human intestinal organoids (HIOs). This complex spheroid consists of an epithelial layer surrounded by a mesenchymal layer containing fibroblasts and smooth muscle cells. Bioreactors in the form of chambers are designed to allow shear stresses to be applied to the apical and basolateral sides of the membrane and permeability is measured over time. Commensal bacteria can be added to the apical tract to simulate the effects of intestinal microbiome and to be observed (Hewes et al., 2020; Pearce et al., 2018; Zhou et al., 2018). This concept seems to be applicable to respiratory tissue engineering although it seems that there are some complexities that need to be adjusted, such as cell type and properties, ECM scaffold used, and dynamic microenvironment systems for microbiome in bioreactors.

\section{Conclusions and Recommendations}

Respiratory tissue has a high complexity based on the anatomical structure of diverse epithelium and has various functions from nasal cavity to alveolar. In addition, knowledge about the role of microbiomes physiologically and pathologically to the mechanism of viral infection is still limited to the identification of microbes found based on metagenomics. The development of in vitro tissue engineering models in the respiratory tract seems to be very necessary given the lack of knowledge about various aspects of the pathophysiology of the respiratory tract. Challenges in this model include achieving a balanced condition between the microenvironment to support the host epithelial tissue and normal microbiome to obtain suitable tissue engineering conditions that are similar in vivo. Research related to modeling that occurs in vitro can represent events that occur in vivo and help determine physiological conditions, the mechanism of the emergence of pathogenic infections, and the development of effective treatment strategies for respiratory diseases in the future.

\section{Novelty Statement}

This review article aims to provide an overview of the various complex mechanisms that occur when infection occurs in the host. Some of them occur due to the role of the microbiome in the respiratory system which has so far been neglected, especially the in vitro infection model using tissue engineering. Thus, the development of an in vitro infection model in respiratory tissue engineering would be more suitable for mimicking the infectious mechanism of the host in the future.

\section{Author's Contribution}

RSK conducts articles with appropriate topics based on recent literature review study, designs and compiles images, also selects included literature. RDA adds to the study literature, revises the image so that it fits, and adds discussion to this article. The final version was approved by all authors.

\section{Conflict of interest}

The authors have declared no conflict of interest.

\section{References}

Abrahao, J.S. and L.B. de Arruda. 2020. Special issue emerging viruses: Surveillance, prevention, evolution, and control. Viruses, 12(3): 306. https://doi.org/10.3390/v12030306

Adachi,A.andT.Miura.2014.AnimalModelstudies 
on viral infections. Front. Microbiol., 5: 672. https://doi.org/10.3389/fmicb.2014.00672

Aghapour, M., P. Raee, S. Moghaddam, P. Hiemstra and I. Heijink. 2018. Airway epithelial barrier dysfunction in chronic obstructive pulmonary disease: Role of cigarette smoke exposure. Am. J. Respir. Cell Mol. Biol., 58(2):157-169. https://doi.org/10.1165/rcmb.2017-0200TR

Alvarado, A. and I. Arce. 2016. Metabolic functions of the lung, disorders and associated pathologies. J. Clin. Med. Res., 8(10): 689-700. https://doi. org/10.14740/jocmr2668w

Barker, E., K. Haverson, C. Stokes, M. Birchall and M. Bailey. 2006. The larynx as an immunological organ: Immunological architecture in the pig as a large animal model. Clin. Exp. Immunol., 143(1): 6-14. https://doi.org/10.1111/j.13652249.2005.02950.x

Barrila, J., A. Crabbe, J. Yang, K. Franco, S. Nydam, R. Forsyth and C. Nickerson. 2018. Modeling host-pathogen interactions in the context of the microenvironment: Three-dimensional cell culture comes of age. Infect. Immun., 86(11). https://doi.org/10.1128/IAI.00282-18

Barthes, J., H. Ozcelik, M. Hindie, A. NdreuHalili, A. Hasan and N. Vrana. 2014. Cell microenvironment engineering and monitoring for tissue engineering and regenerative medicine: The recent advances. Biomed. Res. Int., pp. 921905. https://doi.org/10.1155/2014/921905

Benam, K., S. Dauth, B. Hassell, A. Herland, A. Jain, K. Jang and D. Ingber. 2015. Engineered in vitro disease models. Annu. Rev. Pathol., 10: 195-262. https://doi.org/10.1146/annurevpathol-012414-040418

Binsker, U., J. Lees, A. Hammond and J. Weiser. 2020. Immune exclusion by naturally acquired secretory IgA against pneumococcal pilus-1. J. Clin. Invest., 130(2): 927-941. https://doi. org/10.1172/JCI132005

Brand-Saberi, B. and T. Schafer. 2014. Trachea: Anatomy and physiology. Thorac. Surg. Clin., 24(1): 1-5. https://doi.org/10.1016/j. thorsurg.2013.09.004

Caddeo, S., M. Boffito and S. Sartori. 2017. Tissue engineering approaches in the design of healthy and pathological in vitro tissue models. Front. Bioeng. Biotechnol., 5(40). https://doi. org/10.3389/fbioe.2017.00040

Calle, E.A., K.L. Leiby and M.B. Raredon. 2017. Niklason, LE. Lung regeneration: Steps toward clinical implementation and use. Curr. Opin. Anaesthesiol., 30(1): 23-29. https://doi. org/10.1097/ACO.0000000000000425

Chaicharoenaudomrung, N., P. Kunhorm and P. Noisa. 2019. Three-dimensional cell culture systems as an in vitro platform for cancer and stem cell modeling. World J. Stem. Cells, 11(12): 1065-1083. https://doi.org/10.4252/wjsc.v11. i12.1065

Chanez, P. and A. Bourdin. 2008. Chapter 3 pathophysiology of asthma. In: Castro, M., Kraft, M. (Ed) Clinical Asthma. Philadelphia, Mosby. Pp. 23-34. https://doi.org/10.1016/ B978-032304289-5.10003-7

Crystal, R., S. Randell, J. Engelhardt, J. Voynow and M. Sunday. 2008. Airway epithelial cells: Current concepts and challenges. Proc. Am. Thorac. Soc., 5(7): 772-777. https://doi. org/10.1513/pats.200805-041HR

De Rudder, C., Calatayud, A. Lebeer, S. and T.V de Wiele. 2018. Modelling upper respiratory tract diseases: getting grips on host-microbe interactions in chronic rhinosinusitis using in vitro technologies. Microbiome, 6(1): 75-75. https://doi.org/10.1186/s40168-018-0462-z

de Steenhuijsen, P., E. Sanders and D. Bogaert. 2015. The role of the local microbial ecosystem in respiratory health and disease. Philosophical Trans. R. Soc. Lon. Ser. B Biol. Sci., 370(1675). https://doi.org/10.1098/rstb.2014.0294

Deist, M., R. Gallardo, D. Bunn, T. Kelly, J. Dekkers, H. Zhou and S. Lamont. 2017. Novel mechanisms revealed in the trachea transcriptome of resistant and susceptible chicken lines following infection with newcastle disease virus. Clin. Vaccine Immunol., 24(5). https://doi.org/10.1128/CVI.00027-17

Dhasmana, A., A. Singh and S. Rawal. 2020. Biomedical grafts for tracheal tissue repairing and regeneration. Tracheal tissue engineering: An overview. J.Tissue Eng. Regen. Med., 14: 653-672. https://doi.org/10.1002/ term.3019

Dickson, R., J. Erb-Downward, F. Martinez and G. Huffnagle. 2016. The microbiome and the respiratory tract. Annu. Rev. Physiol., 78: 481-504. https://doi.org/10.1146/annurevphysiol-021115-105238

Eltom, A., G. Zhong and A. Muhammad. 2019. Scaffold techniques and designs in tissue engineering functions and purposes: A review. 
Adv. Mater. Sci. Eng., pp. 3429527. https://doi. org/10.1155/2019/3429527

Estermann, M., C. Bisig, D. Septiadi, A. Petri-Fink and B. Rothen-Rutishauser. 2020. Bioprinting for human respiratory and gastrointestinal in vitro models. Methods Mol. Biol., 2140: 199215. https://doi.org/10.1007/978-1-07160520-2 13

Eugenia, M., D. Patricia, L. Horacio, R. OcadizDelgado and C. Cabello-Gutierrez. 2013. Pathogenesis of viral respiratory infection. In respiratory disease and infection. A new insight. https://doi.org/10.5772/54287

Fernandez, L., S. Norwood and J. Berne. 2008. Chapter 32-tracheal, laryngeal, and oropharyngeal injuries. In: Asensio, J., Trunkey, D. (Ed), Current therapy of trauma and surgical critical care. Philadelphia: Mosby. pp. 215-226. https://doi.org/10.1016/B978-0-323-044189.50036-9

Gao, Z., Y. Kang, J. Yu and L. Ren. 2014. Human pharyngeal microbiome may play a protective role in respiratory tract infections. Genomics Proteomics Bioinf., 12(3): 144-150. https://doi. org/10.1016/j.gpb.2014.06.001

Garcia-Nuñez, M., L. Millares, X. Pomares, R. Ferrari, V. Pérez-Brocal, M. Gallego and E. Monsó. 2014. Severity-related changes of bronchial microbiome in chronic obstructive pulmonary disease. J. Clin. Microbiol., 52(12): 4217-4223. https://doi.org/10.1128/ JCM.01967-14

Gong, H., B. Wang, Y. Shi, Y. Shi, X. Xiao, P. Cao and L. Zhou. 2017. Composition and abundance of microbiota in the pharynx in patients with laryngeal carcinoma and vocal cord polyps. J. Microbiol., 55(8); 648-654. https://doi.org/10.1007/s12275-017-6636-8

Gong, H., Y.Shi, L.Zhou, C.Wu, P.Cao, L.Tao and Y.Wang. 2013. The composition of microbiome in larynx and the throat biodiversity between laryngeal squamous cell carcinoma patients and control population. PLoS One, 8(6). https:// doi.org/10.1371/journal.pone.0066476

Gu, L., H. Deng, Z. Ren, Y. Zhao, S. Yu, Y. Guo and G. Wang. 2019. Dynamic changes in the microbiome and mucosal immune microenvironment of the lower respiratory tract by influenza virus infection. Front. Microbiol., 10(2491). https://doi.org/10.3389/ fmicb.2019.02491
Guan, W., Z. Ni, Y. Hu, W. Liang, C. Ou and J. He. 2020. Clinical characteristics of coronavirus disease 2019 in China. N. Engl. J. Med., 382: 1708-1720. https://doi.org/10.1056/ NEJMoa2002032

Han, M., C. Rajput, T. Ishikawa, C.R. Jarman, J. Lee and M.B. Hershenson. 2018. Small animal models of respiratory viral infection related to asthma. Viruses, 10(12): 682. https://doi. org/10.3390/v10120682

Haschek, W.M., H.R. Witschi and K.J. Nikula. 2002. 28-respiratory system. In: Haschek, W.M., Rousseaux, C.G., Wallig, M.A. (Ed) handbook of toxicologic pathology (Second edition). Academic Press, San Diego. pp. 3-83.

Henkel, J. and D. Hutmacher. 2013. Design and fabrication of scaffold-based tissue engineering. Biol. Nano Mater., 14(3-4): 171. https://doi. org/10.1515/bnm-2013-0021

Hewes, SA., R.L.Wilson, M.K. Estes, N.F. Shroyer, S.E. Blutt and K.J. Grande-Allen. 2020. In vitro models of the small intestine: Engineering challenges and engineering solutions. Tissue Eng. Part B Rev., 26(4): 313-336. https://doi. org/10.1089/ten.teb.2019.0334

Huh, D., D. Leslie, B. Matthews, J. Fraser, S. Jurek, G. Hamilton and D. Ingber. 2012. A human disease model of drug toxicityinduced pulmonary edema in a lung-on-a-chip microdevice. Sci. Transl. Med., 4(159). https:// doi.org/10.1126/scitranslmed.3004249

Huh, D.D., 2015. A human breathing lung on a chip. Ann. Am. Thorac. Soc., 12 Suppl 1(Suppl 1): S42-S44. https://doi.org/10.1513/ AnnalsATS.201410-442MG

Jalili, S., F. Gazzaniga, E. Calamari, D. Camacho, C. Fadel, A. Bein and D. Ingber. 2019. A complex human gut microbiome cultured in an anaerobic intestine on a chip. Nat. Biomed. Eng., 3(7): 520-531. https://doi.org/10.1038/ s41551-019-0397-0

Jaroch, K., A. Jaroch and B. Bojko. 2018. Cell cultures in drug discovery and development: The need of reliable in vitro-in vivo extrapolation for pharmacodynamics and pharmacokinetics assessment. J. Pharm. Biomed. Anal., 147: 297-312. https://doi.org/10.1016/j. jpba.2017.07.023

Kamada, N., G.Y. Chen, N. Inohara and G. Núñez. 2013. Control of pathogens and pathobionts by the gut microbiota. Nat. Immunol., 14(7): 685- 
690. https://doi.org/10.1038/ni.2608

Khazraee, S.P., S.M. Marashi, M. Kaviani and N. Azarpira. 2018. Stem cell-based therapies and tissue engineering of trachea as promising therapeutic methods in mustard gas exposed patients. Int. J. Organ Transplant Med., 9(4): 145-154.

Kiedrowski, M.R., A.E. Paharik, L.W. Ackermann, A.U. Shelton, S.B. Singh, T.D. Starner, A.R. Horswill. 2016. Development of an in vitro colonization model to investigate Staphylococcus aureus interactions with airway epithelia. Cell Microbiol., 18(5): 720-732. https://doi.org/10.1111/cmi.12543

Kim, I.G., S.A. Park, S.H., Lee, J.S. Choi, H. Cho, S.J. Lee and S.K. Kwon. 2020. Transplantation of a 3D-printed tracheal graft combined with iPS cell-derived MSCs and chondrocytes. Sci. Rep., 10(1): 4326. https://doi.org/10.1038/ s41598-020-61405-4

Krause, W.J., 2005. Krause's essential human histology for medical students $3^{\text {rd }}$ Ed. Boca Raton: Universal Publishers.

Kumar, P., N.E. Vrana and A.M. Ghaemmaghami. 2017. Prospects and challenges in engineering functional respiratory epithelium for in vitro and in vivo applications. Microphysiol. Syst., 1(2).https://doi.org/10.21037/mps.2017.09.01

Kumpitsch, C., K. Koskinen, V. Schopf and C. Moissl-Eichinger. 2019. The microbiome of the upper respiratory tract in health and disease. BMC Biol., 17(1): 87.

Lambert, E.K., Y. Hyun, E. Reilly, C. Barilla, S. Gerber, D. Fowell and D.J. Topham. 2016. Live imaging of influenza infection of the trachea reveals dynamic regulation of $\mathrm{CD} 8+\mathrm{T}$ cell motility by antigen. PLoS Pathog., 12(9): e1005881. https://doi.org/10.1371/journal. ppat. 1005881

Lima, M.T., A.C. Andrade, G.P. Oliveira, J.R. Nicoli, F.Martins, E.G. Kroon and J.S.Abrahão. 2019. Virus and microbiota relationships in humans and other mammals: An evolutionary view. Hum. Microbiome J., 11(4). https://doi. org/10.1016/j.humic.2018.11.001

Linden, S.K., P. Sutton, N.G. Karlsson, V. Korolik and M.A. McGuckin. 2008. Mucins in the mucosal barrier to infection. Mucosal Immunol., 1(3): 183-197. https://doi.org/10.1038/ mi.2008.5

Mak, I.W., N. Evaniew and M. Ghert. 2014. Lost in translation: Animal models and clinical trials in cancer treatment. Am. J. Transl. Res., 6(2): 114-118.

Man, W.H., P. de Steenhuijsen, D. Bogaert. 2017. The microbiota of the respiratory tract: Gatekeeper to respiratory health. Nat. Rev. Microbiol., 15(5): 259-270. https://doi. org/10.1038/nrmicro.2017.14

Mandrycky,C.,K.Phong and Y.Zheng.2017.Tissue engineering toward organ-specific regeneration and disease modeling. MRS Commun., 7(3): 332-347. https://doi.org/10.1557/mrc.2017.58

Marrazzo, P., S. Maccari, A. Taddei, L. Bevan, J. Telford, M. Soriani and A. Pezzicoli. 2016. 3D Reconstruction of the human airway mucosa in vitro as an experimental model to study NTHi infections. PLoS One, 11(4). https:// doi.org/10.1371/journal.pone.0153985

Millares, L., G. Bermudo, V. Pérez-Brocal, C. Domingo, M. Garcia-Nuñez, X. Pomares and E. Monsó. 2017. The respiratory microbiome in bronchial mucosa and secretions from severe IgE-mediated asthma patients. BMC Microbiol., 17(1). https://doi.org/10.1186/ s12866-017-0933-6

Navarro, M., J. Ruberte and A. Carretero. 2017. 6-Respiratory apparatus. In: Ruberte, J., Carretero, A., Navarro, M. (Ed) morphological mouse phenotyping: Academic Press, Boston. pp. 147-178. https://doi.org/10.1016/B978-012-812972-2.50006-4

Neto,A.G.,R.A.Hickman,A.Khan,C.Nossa and Z . Pei. 2017. Chapter 1. The upper gastrointestinal tract esophagus and stomach. In: Floch, M.H., Ringel, Y., Allan, W. (Eds) The microbiota in gastrointestinal pathophysiology. Acad. Press, Boston. pp. 1-11. https://doi.org/10.1016/ B978-0-12-804024-9.00001-X

O’Dwyer, D.N., R.P. Dickson and B.B. Moore. 2016. The lung microbiome, immunity, and the pathogenesis of chronic lung disease. J. Immunol., 196(12): 4839-4847. https://doi. org/10.4049/jimmunol.1600279

O'Leary, C., J.L. Gilbert, S. O'Dea, F.J.O'Brien, S.A. Cryan. 2015. Respiratory tissue engineering: Current status and opportunities for the future. Tissue Eng. Part B Rev., 21(4): 323-344. https://doi.org/10.1089/ten.teb.2014.0525

Panara, K. and D. Padalia. 2020. Physiology, swallowing. Treasure island (FL): StatPearls Publishing. 
Panoskaltsis-Mortari, A., 2015. Bioreactor development for lung tissue engineering. Curr. Transplant. Rep., 2(1): 90-97. https://doi. org/10.1007/s40472-014-0048-z

Parker,A.,M.A.E.Lawson,L.Vaux and C.Pin.2018. Host-microbe interaction in the gastrointestinal tract. Environ. Microbiol., 20(7): 2337-2353. https://doi.org/10.1111/1462-2920.13926

Patel, R.G., 2017. Nasal anatomy and function. Facial Plast Surg., 33(1): 3-8. https://doi. org/10.1055/s-0036-1597950

Pearce, S.C., H.G. Coia, J.P. Karl, I.G. PantojaFeliciano, N.C. Zachos and K. Racicot. 2018. Intestinal in vitro and ex vivo models to study host-microbiome interactions and acute stressors. Front. Physiol., 9(1584). https://doi. org/10.3389/fphys.2018.01584

Perez-Losada, M., R.J. Graham, M. Coquillette, A. Jafarey, E. Castro-Nallar, M. Aira and J.M. Mansbach. 2017. The temporal dynamics of the tracheal microbiome in tracheostomised patients with and without lower respiratory infections. PLoS One, 12(8). https://doi. org/10.1371/journal.pone.0182520

Perez-Losada, M., R.J. Graham, M. Coquillette, A. Jafarey, E. Castro-Nallar, M. Aira and J.M. Mansbach. 2018. Tracheal microbiota in patients with a tracheostomy before, during and after an acute respiratory infection. Pediatr. Infect. Dis. J., 37(11): 269-271. https://doi. org/10.1097/INF.0000000000001952

Petersen, T.H., E.A. Calle, M.B. Colehour and L.E. Niklason. 2011. Bioreactor for the long-term culture of lung tissue. Cell Transplant, 20(7): 1117-1126. https://doi. org/10.3727/096368910X544933

Poceviciute, R. and R.F. Ismagilov. 2019. Humangut-microbiome on a chip. Nat. Biomed. Eng., 3(7): 500-501. https://doi.org/10.1038/ s41551-019-0425-0

Poon, C.,M.Zhang,A. Ruys,A.Hong, C. Catuogno and P. Boughton. 2012. A novel dynamic 3-dimensional construct for respiratory tissue engineering. J. Biomimet. Biomater. Tissue Eng., 14: 31-42. https://doi.org/10.4028/www. scientific.net/JBBTE.14.31

Prakash, Y.S., D.J. Tschumperlin and K.R. Stenmark. 2015. Coming to terms with tissue engineering and regenerative medicine in the lung. Am. J. Physiol. Lung Cell. Mol. Physiol., 309(7): 625-638. https://doi.org/10.1152/ ajplung.00204.2015

Proença-Módena, J.L., G.O. Acrani, C.B. Snider and E. Arruda. 2011. Respiratory viral infections. In tropical infectious diseases: principles, pathogens and practice third (ed). Churchill Livingstone, Philadelphia. pp. 378391. https://doi.org/10.1016/B978-0-70203935-5.00058-6

Ramanan, V., M.A. Scull, T.P. Sheahan, C.M. Rice and S.N. Bhatia. 2014. New methods in tissue engineering: Improved models for viral infection. Annu. Rev. Virol., 1: 475499. https://doi.org/10.1146/annurevvirology-031413-085437

Reynolds, S.D., K.E. Pinkerton and A.T. Mariassy. 2015. Chapter 6-epithelial cells of trachea and bronchi. In: Parent, R.A. (Ed) comparative biology of the normal lung (Second Edition). Acad. Press, San Diego. pp. 61-81. https://doi. org/10.1016/B978-0-12-404577-4.00006-0

Roomans, G.M., 2010. Tissue engineering and the use of stem/progenitor cells for airway epithelium repair. Eur. Cell. Mater., 19: 284299. https://doi.org/10.22203/eCM.v019a27

Saji, J., M. Tebogo and M. Ntwasa. 2019. TwoDimensional (2D) and Three-Dimensional (3D) Cell Culturing in Drug Discovery. Cell Culture Intech Open.

Scherzad, A., R. Hagen and S. Hackenberg. 2019. Current understanding of nasal epithelial cell mis-differentiation. J. Inflamm.Res., 12: 309317. https://doi.org/10.2147/JIR.S180853

Selden, C. and B. Fuller. 2018. Role of bioreactor technology in tissue engineering for clinical use and therapeutic target design. Bioengineering (Basel), 5(2). https://doi.org/10.3390/ bioengineering 5020032

Siegel, S.J. and J.N. Weiser. 2015. Mechanisms of bacterial colonization of the respiratory tract. Annu. Rev. Microbiol., 69: 425444. https://doi.org/10.1146/annurevmicro-091014-104209

Starner, T.D., N. Zhang, G. Kim, M.A. Apicella and P.B. McCray. 2006. Haemophilus influenzae forms biofilms on airway epithelia: Implications in cystic fibrosis. Am. J. Respir. Crit. Care Med., 174(2): 213-220. https://doi.org/10.1164/ rccm.200509-1459OC

Sudhakara, P., I. Sellamuthu and A.W. Aruni. 2019. Bacterial sialoglycosidases in virulence and pathogenesis. Pathogens (Basel, 
Switzerland). 8(1)39. https://doi.org/10.3390/ pathogens 8010039

Swearengen, J.R., 2018. Choosing the right animal model for infectious disease research. Anim. Model Exp. Med., 1(2): 100-108. https://doi. org/10.1002/ame2.12020

Taneja, V.D., 2017. Microbiome: Impact of gender on function and characteristics of gut microbiome. In: Legato, M.J. (Ed) principles of gender-specific medicine: Gender in the genomic era: Third Edition. Academic Press, San Diego. pp. 569-583. https://doi.org/10.1016/B978-012-803506-1.00027-9

Taubenberger, J.K. and D.M. Morens. 2008. The pathologyofinfluenzavirusinfections.Annu.Rev. Pathol., 3: 499-522. https://doi.org/10.1146/ annurev.pathmechdis.3.121806.154316

Tebyanian, H., A. Karami, M.R. Nourani, E. Motavallian, A. Barkhordari, M. Yazdanian and A. Seifalian. 2019. Lung tissue engineering: An update. J. Cell Physiol., 234(11): 19256-19270. https://doi.org/10.1002/jcp.28558

Thiebes, A.L., S. Albers, C. Klopsch, S. Jockenhoevel and C.G. Cornelissen. 2015. Spraying respiratory epithelial cells to coat tissue-engineered constructs. Biores. Open Access, 4(1): 278-287. https://doi.org/10.1089/ biores.2015.0015

Troy, N.M. and A. Bosco. 2016. Respiratory viral infections and host responses; insights from genomics. Respir. Res., 17(1): 156. https://doi. org/10.1186/s12931-016-0474-9

Wang,H.,W.Dai,X.Feng,Q.Zhou,H.Wang,Y.Yang and Y. Zheng. 2018. Microbiota composition in upper respiratory tracts of healthy children in Shenzhen, China, differed with respiratory sites and ages. Biomed. Res. Int., pp. 6515670. https://doi.org/10.1155/2018/6515670

Wang, L., G. Yin, Y. Guo, Y. Zhao, M. Zhao, Y.
Lai and Z. Huang. 2019. Variations in oral microbiota composition are associated with a risk of throat cancer. Front Cell Infect. Microbiol., 9(205). https://doi.org/10.3389/ fcimb.2019.00205

Wilks, J., H. Beilinson and T.V. Golovkina. 2013. Dual role of commensal bacteria in viral infections. Immunol. Rev., 255(1): 222-229. https://doi.org/10.1111/imr.12097

Yoo, J.K., T.S. Kim, M.M. Hufford and T.J. Braciale. 2013. Viral infection of the lung: host response and sequelae. J. Allergy Clin. Immunol., 132(6):1263-1276. https://doi.org/10.1016/j. jaci.2013.06.006

Yuksel, H. and A. Turkeli. 2017. Airway epithelial barrier dysfunction in the pathogenesis and prognosis of respiratory tract diseases in childhood and adulthood. Tissue Barriers, 5(4). https://doi.org/10.1080/21688370.2017.1367 458

Zachary,J. and M.McGavin. 2017. Pathologic basis of veterinary disease expert consult. In: Elsevier, Missouri, USA.

Zanin, M., P. Baviskar, R. Webster and R. Webby. 2016. The interaction between respiratory pathogens and mucus. Cell Host Microbe, 19(2): 159-168. https://doi.org/10.1016/j. chom.2016.01.001

Zhou, F., T.Yu, R. Du, G. Fan, Y. Liu, Z. Liu and B. Cao. 2020. Clinical course and risk factors for mortality of adult inpatients with COVID-19 in Wuhan, China: A retrospective cohort study. The Lancet, 395:1054-1062. https://doi. org/10.1016/S0140-6736(20)30566-3

Zhou, W., Y. Chen, T. Roh, Y. Lin, S. Ling, S. Zhao and D. Kaplan. 2018. Multifunctional bioreactor system for human intestine tissues. ACS Biomater. Sci. Eng. 4(1):231-239. https:// doi.org/10.1021/acsbiomaterials.7b00794 